\title{
Editorial
}

\section{Circulating biomarkers for cardiovascular diseases: the beats never stop}

\author{
Lei $\mathrm{XI}^{1, *}$, George KOUVELOS ${ }^{2, *}$, Nazareno PAOLOCCI ${ }^{3, *}$ \\ ${ }^{1}$ Pauley Heart Center, Department of Internal Medicine, Virginia Commonwealth University, Richmond, VA 23298-0204, USA; \\ ${ }^{2}$ Department of Vascular Surgery, University of Thessaly, Larissa, Greece; ${ }^{3}$ Heart \& Vascular Institute, Johns Hopkins University, \\ Baltimore, MD 21205, USA
}

Acta Pharmacologica Sinica (2018) 39: 1065-1067; doi: 10.1038/aps.2018.43; published online 21 Jun 2018

Cardiovascular diseases (CVD) remain a major health issue and a grave socio-economic burden, being the No. 1 killer in major industrial countries and the main reason for frequent rehospitalization $^{[1]}$. In recent years, unmet needs for a distinct cardiovascular risk assessment allowing for standardized guidelines on personalized therapy have led to an intensive research on biomarkers. Even in this post-cTn (cardiac troponin) and post-BNP (B-type natriuretic peptide) era, there are still significant gaps and huge demands for more reliable and innovative methods and tools for diagnosis and evidenceguided management of CVD. Under this context, the basic research and clinical use of biomarkers have gained a tremendous momentum in the past decade. The use of single or multiple biomarkers as reliable and reproducible indicatives of the risk, severity, and progression stage of CVD greatly enhances the diagnostic decision and prognostic ability of emergency department physicians, cardiologists, and cardiovascular surgeons in their routine patient care duties. The prudential utilization of valid biomarkers in clinical practice can reduce time and costs for an accurate diagnosis and proper personalized therapy of CVD patients. As suggested in Figure 1, biomarker testing may play a central role in the evidence-based Cardiovascular Medicine and it can in turn promote the improved clinical outcome, better quality of life, and alleviated socioeconomic burden of CVD.

Accordingly, this Special Issue of Acta Pharmacologica Sinica was planned to provide a forum for high quality original research and review articles to showcase the most recent advances in translational research and clinical practices on a wide-spectrum array of conventional as well as novel cardio-

\footnotetext{
${ }^{*}$ To whom correspondence should be addressed. E-mail lei.xi@vcuhealth.org (Lei XI) geokouv@gmail.com (George KOUVELOS) npaoloc1@jhmi.edu (Nazareno PAOLOCCI)
}

vascular biomarkers. Coverage of this Special Issue encompasses various forms of large or small molecules, such as proteins, peptides, cytokines, microRNAs, and metabolic by-

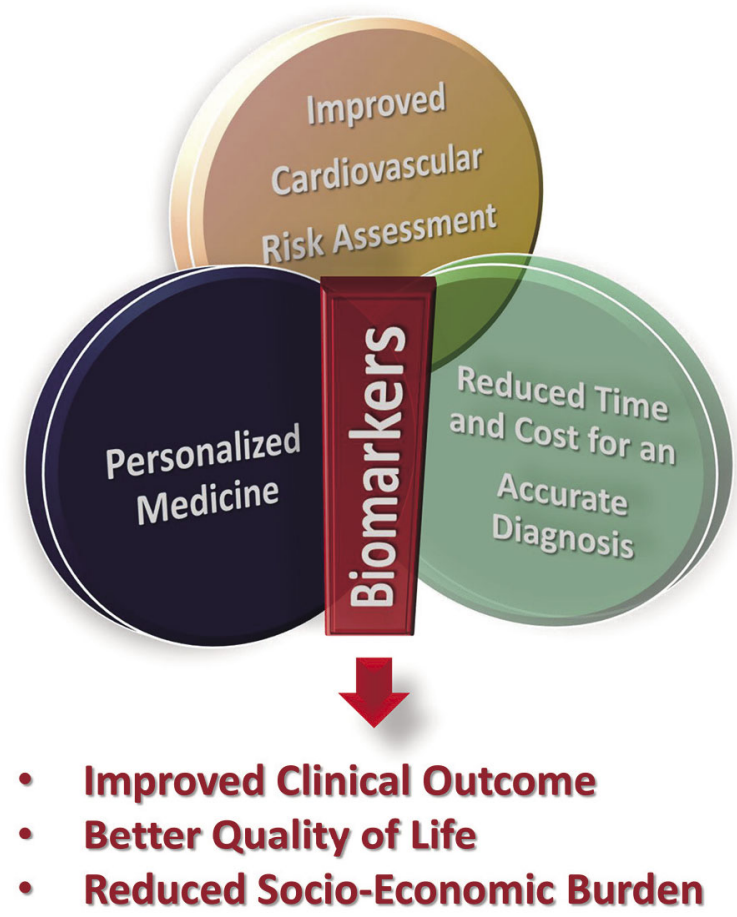

Figure 1. A graphical notion suggesting biomarker testing may play a central role in improvement of cardiovascular risk assessment and reduced time and cost for an accurate diagnosis and personalized medicine that can eventually lead to improved clinical outcome, better quality of life, and alleviated socioeconomic burden of cardiovascular diseases. 
products that are released from injured cardiovascular tissues into blood stream during various CVD events.

A total of 18 papers authored by 108 scientists from 15 countries, i.e. Australia, Austria, China, Czech Republic, Germany, Greece, Hong Kong (China), Malaysia, Poland, Portugal, Romania, Slovak Republic, Switzerland, United Kingdom, and United States, are accepted and represent a truly global effort for tackling the unsolved issues and new discoveries of CVD biomarkers. These collective efforts have also made the current Special Issue probably the most "international" volume of Acta Pharmacologica Sinica in the Journal's 30+-year history.

This Special Issue covers a variety of pathological types of CVD, including acute myocardial infarction (AMI ${ }^{[2-5]}$, heart failure $^{[6]}$, aortic aneurysms ${ }^{[7,8]}$, carotid restenosis ${ }^{[9]}$, pulmonary arterial hypertension $(\mathrm{PAH})^{[10]}$, and cardiac amyloido$\operatorname{sis}^{[11]}$. Several review articles provided in-depth analyses on the important diagnostic and prognostic roles of zinc ${ }^{[12]}$, oxylipins ${ }^{[13]}$, and leptin ${ }^{[14]}$ in the fundamental cellular signaling and pathogenic processes in CVD and diabetes. Notably, all the 7 original research articles are clinical research on Asian patients with $\mathrm{AMI}^{[5]}$ or $\mathrm{PAH}^{[10]}$ as well as European patients with AMI ${ }^{[2]}$, chronic heart failure ${ }^{[6]}$, vascular aneurysm ${ }^{[7]}$ or restenosis ${ }^{[9]}$, and stroke ${ }^{[15]}$.

It is noteworthy that 4 review articles ${ }^{[4,16-18]}$ and 2 original research papers ${ }^{[2,10]}$ in this Special Issue are focusing on a cutting-edged topic concerning the potential roles of circulating noncoding RNAs (ncRNAs), which include microRNAs (miRNAs), long noncoding RNAs (lncRNAs) and circular RNAs (circRNAs), in serving as a new generation of biomarkers for CVD. Among these elegant works, Bayoumi et al. reviewed the circular noncoding RNAs as potential therapies and circulating biomarkers for $\mathrm{CVD}^{[18]}$. The similar topic was also thoroughly analyzed by Zhou et al ${ }^{[17]}$ and Stepien et al ${ }^{[16]}$, who provided their own perspectives. Two other papers by Wang et $a l^{[4]}$ and Song et $a l^{[10]}$ provided more focused discussions respectively on the use of microRNAs for early detection of CVD such as $\mathrm{AMI}^{[4]}$ and pulmonary hypertension ${ }^{[10]}$. We wish this panel of 6 articles may help the readers to gain a comprehensive grasp on the upcoming trend of using miRNAs, IncRNAs, and circRNAs as the newest class of CVD biomarkers that will be eventually utilized in the clinical settings considering the above-discussed keen interests and intensive investigations in this research area.

Another highlighted area of discussion in this Special Issue is on the technological and methodological innovations for the CVD biomarker detection. Our invited experts have communicated their original research results using various technologies from advanced chip-based digital $\mathrm{PCR}^{[2]}$ to $2 \mathrm{D}$-gel analysis $^{[5]}$. They also thoroughly reasoned the needs for multiplex approach to enhance diagnostic accuracy superior to the single biomarker detection ${ }^{[19]}$.

In brief, we believe that these collective efforts will be instrumental for future research and technological development of new CVD biomarkers that can be used for saving lives and improving clinical outcomes in tens of millions CVD patients worldwide. For these meaningful endeavor and noble cause, we would like to most sincerely thank all of our participating authors and numerous contributing expert reviewers, as well as the Editor-in-Chief (Dr Jian DING), Editorial Manager (Ms. Min-shu WU), Executive Editor (Ms. Qian-rong ZHU), and Handling Editor (Dr Bin-jia ZHANG) and other journal staff for their extraordinary efforts and supports that jointly made this extensive publication endeavor possible.

\section{References}

1 Roth GA, Johnson C, Abajobir A, Abd-Allah F, Abera SF, Abyu G, et al. Global, Regional, and National Burden of Cardiovascular Diseases for 10 Causes, 1990 to 2015. J Am Coll Cardiol 2017; 70: 1-25.

2 Robinson S, Follo M, Haenel D, Mauler M, Stallmann D, Heger LA, et al. Chip-based digital PCR as a novel detection method for quantifying microRNAs in acute myocardial infarction patients. Acta Pharmacol Sin 2018; 39: 1217-27.

3 Ye XD, He Y, Wang S, Wong GT, Irwin MG, Xia Z. Heart-type fatty acid binding protein (H-FABP) as a biomarker for acute myocardial injury and long-term post-ischemic prognosis. Acta Pharmacol Sin 2018; 39: 1155-63.

4 Wang $\mathrm{C}$, Jing Q. Non-coding RNAs as biomarkers for acute myocardial infarction. Acta Pharmacol Sin 2018; 39: 1110-9.

5 Rahim MAA, Rahim ZHA, Ahmad WAW, Bakri MM, Ismail MD, Hashim $\mathrm{OH}$. Inverse changes in plasma tetranectin and titin levels in patients with type 2 diabetes mellitus: a potential predictor of acute myocardial infarction? Acta Pharmacol Sin 2018; 39: 1197-207.

6 Jirak P, Fejzic D, Paar V, Wernly B, Pistulli R, Rohm I, et al. Influences of Ivabradine treatment on serum levels of cardiac biomarkers SST2, GDF-15, suPAR and H-FABP in patients with chronic heart failure. Acta Pharmacol Sin 2018; 39: 1189-96.

7 Papoutsis K, Kapelouzou A, Tsilimigras DI, Patelis N, Kouvelos G, Schizas $\mathrm{D}$, et al. Associations between serum relaxin 2, aneurysm formation/size and severity of atherosclerosis: a preliminary prospective analysis. Acta Pharmacol Sin 2018; 39: 1243-8.

8 Tsilimigras DI, Sigala F, Karaolanis G, Ntanasis-Stathopoulos I, Spartalis E, Spartalis M, et al. Cytokines as biomarkers of inflammatory response after open versus endovascular repair of abdominal aortic aneurysms: a systematic review. Acta Pharmacol Sin 2018; 39: 1164-75.

9 Maskanakis A, Patelis N, Karaolanis G, Davakis S, Schizas D, Perrea D, et al. Apolipoprotein $\mathrm{J}$ as a predictive biomarker for restenosis after carotid endarterectomy: a retrospective study. Acta Pharmacol Sin 2018; 39: 1237-42.

10 Song XW, Zou LL, Cui L, Li SH, Qin YW, Zhao XX, et al. Plasma miR451 with echocardiography serves as a diagnostic reference for pulmonary hypertension. Acta Pharmacol Sin 2018; 39: 1208-16.

11 Luciani M, Troncone L, del Monte F. Current and future circulating biomarkers for cardiac amyloidosis. Acta Pharmacol Sin 2018; 39: 1133-41.

12 Choi S, Liu X, Pan Z. Zinc deficiency and cellular oxidative stress: prognostic implications in cardiovascular diseases. Acta Pharmacol Sin 2018; 39: 1120-32.

13 Nayeem MA. Role of oxylipins in cardiovascular diseases. Acta Pharmacol Sin 2018; 39: 1142-54.

14 Katsiki N, Mikhailidis DP, Banach M. Leptin, cardiovascular diseases and type 2 diabetes mellitus. Acta Pharmacol Sin 2018; 39: 117688.

15 Hutanu A, lancu M, Balasa R, Maier S, Dobreanu M. Predicting functional outcome of ischemic stroke patients in Romania based on plasma CRP, sTNFR-1, D-Dimers, NGAL and NSE measured using a 
biochip array. Acta Pharmacol Sin 2018; 39: 1228-36.

16 Stepien E, Costa MC, Kurc S, Drozdz A, Cortez-Dias N, Enguita FJ. The circulating non-coding RNA landscape for biomarker research: lessons and prospects from cardiovascular diseases. Acta Pharmacol Sin 2018; 39: 1085-99.

17 Zhou SS, Jin JP, Wang JQ, Zhang ZG, Freedman JH, Zheng Y, et al. miRNAs in cardiovascular diseases: potential biomarkers, therapeutic targets and challenges. Acta Pharmacol Sin 2018; 39: 1073-84.

18 Bayoumi AS, Aonuma T, Teoh JP, Tang YL, Kim IM. Circular noncoding RNAs as potential therapies and circulating biomarkers for cardiovascular diseases. Acta Pharmacol Sin 2018; 39: 1100-9.

19 Adamcova M, Simko F. Multiplex biomarker approach to cardiovascular diseases. Acta Pharmacol Sin 2018; 39: 1068-72. 